\title{
Random Polymers and Generalized Urn Processes
}

\author{
Simone Franchini and Riccardo Balzan \\ Sapienza Università di Roma, 1 Piazza Aldo Moro, 00185 Roma, Italy
}

We describe a microcanonical approach for polymer models that combines atmospheric methods with urn theory. We show that Large Deviation Properties of urn models can provide quite deep mathematical insight by analyzing the Random Walk Range problem in $\mathbb{Z}^{d}$. We also provide a new mean field theory for the Range Problem that is exactly solvable by analogy with the Bagchi-Pal urn model. 


\section{INTRODUCTION}

In this paper we present a novel approach to deal with microcanonical polymer models derived in analogy with urn process theory. The main point in this method is that it is possible to relate the density of states of an interacting chain with the problem of computing the large deviation behavior of an associated Markov urn process [1-3] once the urn function of the problem is identified (see below). Here we deal with models that can be related to a two-color urn, first introduced by Hill, Lane and Sudderth [4-7, for which a detailed Large deviations theory has been recently developed [7]. In particular, we will provide an explicit example by studying the classic Random Walk range problem (RP, [8 12]), that is, computing the number of different lattice sites visited by a random walk of given lenght.

The HLS urns is a Markov process first introduced in [4. Consider an infinite capacity urn with a finite number of black and white balls and let $y_{t}$ the fraction of black balls inside the urn at a certain time $t$ of the evolution, then in a HLS process of urn function $\pi(y)$ at each step a black ball is added with probability $\pi\left(y_{t}\right)$ and a white one is added otherwise. The process is then parametrized by the function $\pi(y)$, that represent the probability of adding a black ball at the considered step conditioned that the urn has reached a certain fraction of black balls.

The second ingredient is the endpoint atmosphere, introduced some years ago within the study of the Self-Avoiding Walk as the number of ways in which a chain of $N$ steps can be continued by adding one monomer to the endpoint [13. As we shall see, it is possible to combine this two ideas together and define HLS processes that converge to a given polymer model in the thermodynamic limit by interpreting the probability that adding a step to a given chain produces an increase in energy analog to adding a black ball in the the associated urn process, ie the number of black balls will represent the total energy of our polymer.

Before starting let introduce some notation. Let $\mathbb{L}$ be some regular lattice and let $L_{1}$ be the possible orientations on $\mathbb{L}$. Then we call a chain $\omega_{N} \in L_{1}^{N}$ of $N$ steps on $\mathbb{L}$ the ordered -sequence of steps $\delta x_{t} \in L_{1}$ for $1 \leq t \leq N$, with $L_{1}^{N}$ the set of distinct random walks of $N$ steps on $\mathbb{L}$, thus $\omega_{N}=\left\{\delta x_{1}, \ldots, \delta x_{N}\right\}$. If we fix the starting point $x_{0}$ we can also represent $\omega_{N}$ by the positions $x_{t} \in \mathbb{L}$, related to the steps $\delta x_{t}$ by $\delta x_{t}=x_{t}-x_{t-1}$. Hereafter we will assume that $x_{0} \equiv 0$ and

$$
\omega_{N}=\left\{x_{0}, x_{1}, \ldots, x_{N}\right\}
$$

with steps $x_{t}-x_{t-1} \in L_{1}$ for all times $1 \leq t \leq N$.

Now consider the interaction energy $H\left(\omega_{N}\right)$, that is the energy associated to the chain configuration $\omega_{N}$. We assume that the interaction energy $H$ can be defined for arbitrary size $N$ of the walks. In general, we can define the free energy density per monomer of the interaction $H$ supported by $L_{1}^{N}$ in the thermodynamic limit

$$
f(\beta)=-\lim _{N \rightarrow \infty} \frac{1}{\beta N} \log \sum_{\omega_{N} \in L_{1}^{N}} e^{-\beta H\left(\omega_{N}\right)} .
$$

After rescaling by the number of possible walks we can write $-\beta f(\beta)=\log \left|L_{1}\right|+\zeta(\beta)$ where $\zeta(\beta)$ is the Cumulant Generating Function (CGF) of the variable $H\left(\omega_{N}\right)$

$$
\zeta(\beta)=\lim _{N \rightarrow \infty} \frac{1}{N} \log \left\langle e^{-\beta H\left(\omega_{N}\right)}\right\rangle_{L_{1}^{N}}
$$

with average over $\omega_{N}$ taken uniform on $L_{1}^{N}$.

Then, let $\omega_{N}$ be a random chain of $N$ step and define the sequence $\omega_{t} \subset \omega_{N}$ subwalks of $\omega_{N}$ according to the monomer ordering $t$, ie $\omega_{t}=\left\{x_{0}, \ldots, x_{t}\right\}$. In this paper we will deal with energy functions that satisfy

$$
H\left(\omega_{t+1}\right)-H\left(\omega_{t}\right) \in\{0,1\}
$$

for all $\omega_{N} \in L_{1}^{N}$ and all $t$. This condition ensures that the energy can either increase of one unit or do not increase at all when a monomer is added to the endpoint of $\omega_{N}$, and is an important technical point to connect with the HLS urns as it allows to directly identify an increase in energy followed by one step grows with adding a black ball to the associated urn. It is possible to generalize to include more general transition spectra (multicolor urns) but here we consider the binary cases as the LDP for such urns have been already developed in detail [7].

\section{HLS URNS}

Before going further we need to introduce the HLS process [4 7] and sketch some of its main properties we will use in the following. An HLS urn is a two color urn process that is governed by a functional parameter $\pi(y)$ called urn 
function [7. Let us consider an infinite capacity urn containing two kinds of elements, say black and white balls, and denote by

$$
Y=\left\{Y_{t_{0}}, Y_{t_{0}+1}, \ldots, Y_{N}\right\}
$$

the process describing the number of black balls inside the urn during its evolution from $t=t_{0}$ to $N$. The process $Y$ evolves as follows, let $y_{t}=Y_{t} / t$ be the fraction of black balls at time $t$, then at step $t+1$ a new ball is added, whose color is black with probability $\pi\left(y_{t}\right)$ and white with probability $1-\pi\left(y_{t}\right)$.

Then, let $Y$ be an HLS urn process stopped at $N$, with initial condition $Y_{t_{0}}=M_{0}$, describing the number of black balls in the evolution of a HLS urn of urn function $\pi$. By simple arguments on conditional expectations it is not hard to prove that the process satisfy the following master equation

$$
P\left(Y_{N+1}=M+1\right)=\pi\left(\frac{M}{N}\right) P\left(Y_{N}=M\right)+\left(1-\pi\left(\frac{M+1}{N}\right)\right) P\left(Y_{N}=M+1\right)
$$

that can be iterated backward to the initial condition

$$
P\left(Y_{t_{0}}=M_{0}\right)=I\left(Y_{t_{0}}=M_{0}\right),
$$

where $\pi$ is the urn function and $I\left(Y_{t_{0}}=M_{0}\right)$ is indicator function, valued one if $Y_{t_{0}}=M_{0}$ and zero otherwise.

In [7] the cumulant generating function of the process

$$
\zeta(\beta)=\lim _{N \rightarrow \infty} \frac{1}{N} \log \sum_{k \leq N} e^{-\beta k} P\left(Y_{N}=k\right)
$$

is studied in detail and it is proven that it must satisfy the following nonlinear differential equation

$$
\partial_{\beta} \zeta(\beta)=\pi^{-1}\left(\frac{e^{\zeta(\beta)}-1}{e^{\beta}-1}\right)
$$

with $\pi^{-1}$ inverse urn function. Of special interest for our scopes will be the case of linear urn functions

$$
\pi(y)=a+b y
$$

that in [7] are shown to be equivalent to the Baghi-Pal model [3, 6], a widely investigated model due to its relevance in studying branching phenomena and random trees (see [1 3] for some reviews). Linear urn functions satisfy the differential equation

$$
\partial_{\beta} \zeta(\beta)=-\frac{a}{b}+\frac{1}{b}\left(\frac{e^{\zeta(\beta)}-1}{e^{\beta}-1}\right)
$$

The above equation can be integrated exactly. Although the solution depends on the considered parameter region, for our analysis it will suffice to take $a>0, a+b<1$ and $b>0, \beta>0$. From Corollary 10 of [7] we have that

$$
1-e^{-\zeta(\beta)}=\frac{a}{b} e^{-\frac{a}{b} \beta}\left(1-e^{-\beta}\right)^{\frac{1}{b}} B\left(\frac{a}{b}, \frac{b-1}{b} ; 1-e^{-\beta}, 1\right)
$$

where $B(q, p ; u, v)$ is a Generalized Hypergeometric function of the second kind

$$
B(q, p ; u, v)=\int_{u}^{v} d t(1-t)^{q-1} t^{p-1} .
$$

As we shall see in short our mean field theory will be described by linear urn theory above.

\section{URN ANALOGY}

Although the limitations imposed by Eq. (4), simple two colors HLS urns still allows to describe interesting models (that are not limited to polymer physics). The problem we investigate here is the the Random Walk Range problem (RP) on the cubic lattice $\mathbb{Z}^{d}$ [9, 10, 12, a model showing [12] a full crossover from Self-Avoiding Walks (SAW) [17] to collapsed globular configuration in the range density per monomer, and is shown to have an interesting geometric Coil-to-Globule transition (CG, the chain collapses from an extended random coil to a liquid-like cluster, [14, 15]) at a critical range density for any $d \geq 3$. 


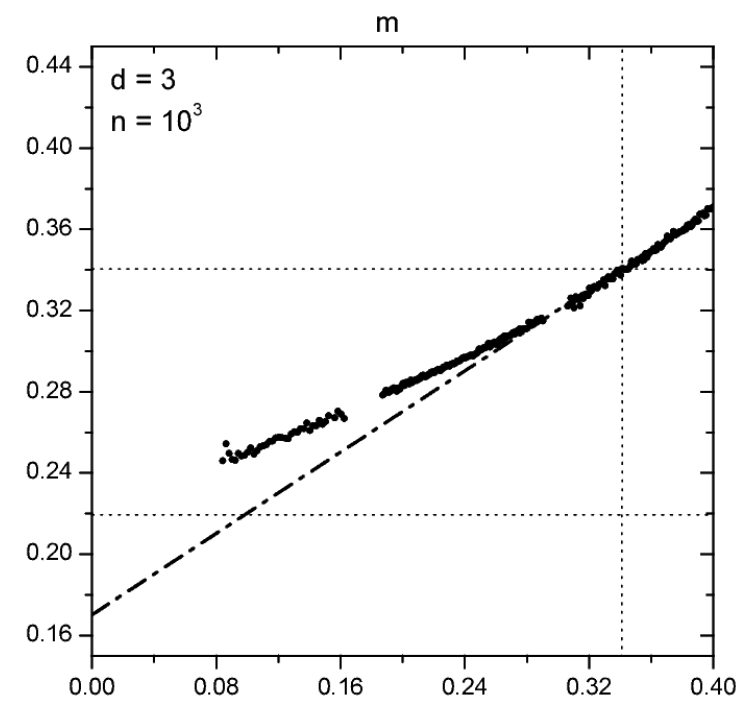

Figure 1. Numerical estimate of $\pi_{N}(\lfloor N m\rfloor)$ from Eq. (17), for dimensions $d=3$ and $0 \leq m \leq 0.4$. The lengths of the chains were $N=1000$. The vertical dot line and the upper horizontal dot line show the Polya RW constant $C_{3}$ [16], and crosses at the RW point. The lower horizontal dotted line is the limit $\pi_{\infty}(0)=\eta_{3}$ [17, 18, according to [19. The dash dotted line is the MF aproximation $\pi_{M F}(m)=\left(C_{3}+m\right) / 2$, computed according to Eq. 28] and [29].

Take a walk $\omega_{N} \in L_{1}^{N}$ and define the number of different sites of $\mathbb{L}$ visited by $\omega_{N}$. We will approach the RP by studying interaction energy

$$
H\left(\omega_{N}\right)=N-R\left(\omega_{N}\right)=N-\sum_{x \in \mathbb{Z}^{d}} I\left(x \in \omega_{N}\right)
$$

an Hamiltonian first introduced by Stanley et Al. in [11, 23]. To show the urn process analogy we first need to introduce some microcanonical estimators. Let

$$
L_{N}(M)=\left\{\omega_{N} \in L_{1}^{N}: H\left(\omega_{N}\right)=M\right\}
$$

be the fraction of walks of length $N$ with an energy of exactly $M$, then call $P\left(H\left(\omega_{N}\right)=M\right)$ the probability that a chain $\omega_{N}$ uniformly picked from $L_{N}$ has energy $M$. Notice that the constraint of binary energy increase guarantees that, for all these functions, $m$ is a real parameter between zero and one. Then, let consider a walk of $N$ steps $\omega_{N} \in L_{1}^{N}$ and define the average of the energy after a random continuation $\omega_{1}^{*} \in L_{1}$ from the endpoint of $\omega_{N}$

$$
\delta H_{1}\left(\omega_{N}\right)=\left\langle H\left(\omega_{N} \cup \omega_{1}^{*}\right)-H\left(\omega_{N}\right)\right\rangle_{L_{1}}=\frac{1}{\left|L_{1}\right|} \sum_{x \in \mathbb{Z}^{d}} I\left(x \in \omega_{N}\right) I\left(x \in L_{1}\right)
$$

Since energy can increase only by zero or one then the average increase $\delta H_{1}\left(\omega_{N}\right)$ equals the probability that a random continuation of the walk $\omega_{N}$ from its endpoint $x_{N}$ produces a self interaction according to $H$. We then define the atmosphere

$$
\pi_{N}(M)=\left\langle\delta H_{1}\left(\omega_{N}\right)\right\rangle_{L_{N}(M)},
$$

that is the probability of self-intersection after a random continuation of $\omega_{N}$, conditioned to the event that the range is $R\left(\omega_{N}\right)=N-M$.

It can be proven that $P\left(H\left(\omega_{N}\right)=M\right)$ satisfy the following Master equation

$$
P\left(H\left(\omega_{N+1}\right)=M\right)=\pi_{N}(M) P\left(H\left(\omega_{N}\right)=M\right)+\left(1-\pi_{N}(M+1)\right) P\left(H\left(\omega_{N}\right)=M+1\right)
$$

with initial condition

$$
P\left(H\left(\omega_{1}\right)=M\right)=I(M=0) .
$$

If we take $H\left(\omega_{N}\right)=Y_{N}$ it is clear that the Master Equation for the measure of the event is the same of the event $Y_{N}=M$ of an HLS urn of non-homogeneous urn function $\pi_{N}(M)$. In [7] it is shown that if

$$
\lim _{N \rightarrow \infty}\left|\pi_{N}(\lfloor N m\rfloor)-\pi(m)\right|=0
$$


then the cumulant generating function of the process is the same of an HLS urn of urn function $\pi(m)$. The existence of $\pi(m)$ for the RP can be inferred by subadditivity, but we do not give a proof here because the convergence of $\pi_{N}(\lfloor N m\rfloor)$ toward some smooth $\pi(m)$ is already clear from our numerical analysis (see Figure 3 ).

\section{NUMERICAL RESULTS}

In Figures 1,2 and 3 we present our numerical results concerning the urn function $\pi_{N}(M)$ associated to the RP on $\mathbb{Z}^{d}, 3 \leq d \leq 6$, for which some properties can be deduced also from known results in Random and Self-Avoiding Walks theory [9, 17, 18].

The numerical simulations where performed by a standard implementation of the Pruned-Enriched Rosenbluth Method, PERM, see [24 27]. For $3 \leq d \leq 6$ we restricted our attention to the region $M / N<m_{c}$, where the typical configuration of $\omega_{N}$ is supposed to be in the universality class of the self-avoiding walk.

In a previous paper [12] we numerically studied the event $H\left(\omega_{N}\right)=\lfloor m N\rfloor$ and found a CG transition for some critical value $m=m_{c} \in(0,1)$. We studied the critical exponent governing the mean square displacement,

$$
\nu_{d}(m)=\lim _{N \rightarrow \infty} \frac{\log \left\langle x_{N}^{2}\right\rangle_{C_{N}(\lfloor N m\rfloor)}}{2 \log N},
$$

concluding the above limit exists and $\nu_{d}\left(m<m_{c}\right)=\nu_{d}, \nu_{d}\left(m=m_{c}\right)=\nu_{c}, \nu_{d}\left(m>m_{c}\right)=1 / d$, where $\nu_{d}$ is the critical exponent governing the end-to-end distance of the Self-Avoiding Walk [17]. Also, $\nu_{c}=1 / 2, m_{c}=C_{d}$ Polya constant [16] for $d=3,4$, and that for $d \geq 5$ it is expected that $m_{c}>C_{d}$ (see [12 for further details about this topics).

Here we observe that $\pi_{N}(M)$ approaches to some continuous $\pi(M / N)$ uniformly on the considered range. Quite surprisingly, we also observe that for $d \geq 4$ the function $\pi$ suddenly approaches some linear function (see Figures 2 and 3)

$$
\pi_{M F}(m)=a_{R P}+b_{R P} m
$$

in the region $0 \leq m \leq m_{c}$. Assuming a linear urn function, the coefficients can be computed exactly from RW theory by relating them to the variance of the energy $\sigma_{d}^{2}$.

The constant $\sigma_{d}$ can be computed from Jain-Pruitt theorem on the variance of the RP ([9, 28, 231, see also [32] for an explicit computation). For $d=3$, Jain and Pruitt have shown [9, 28, 29] that the leading order of the variance of $R\left(\omega_{N}\right)$ for a random walk is $\sigma_{3}^{2} N \log (N)$ with $\sigma_{3}$ exactly computable, while for for $d \geq 4$ the same authors shows that the variance is $\sigma_{d}^{2} N$ with $\sigma_{d}$ expressed by the relation

$$
\sigma_{d}^{2}=C_{d}\left(1-C_{d}\right)+2 a
$$

Accurate estimates for $C_{d}$ are in [16]. To determine $a$ we follow [9, 18, 28, 29, 32]. Let first introduce the propagator

$$
G(x)=\int_{[-\pi, \pi]^{d}} \frac{d q}{(2 \pi)^{d}} e^{i q x}\left(1-\tilde{\lambda}_{d}(q)\right)^{-1},
$$

where $\tilde{\lambda}_{d}(q), q \in[-\pi, \pi]^{d}$ is the structure factor of the hypercubic lattice $\mathbb{Z}^{d}$

$$
\tilde{\lambda}_{d}(q)=\frac{1}{d} \sum_{i=1}^{d} \cos \left(q_{i}\right)
$$

and where $q_{i}$ are the components of the dual vector $q$. The quantity $G(x)$ represents the expected number of visits to a given site $x \in \mathbb{Z}^{d}$ for an infinite lenght random walk. From standard random walks theory follows 9

$$
\frac{C_{d}}{1-C_{d}}=\sum_{x \in \mathbb{Z}^{d} \backslash\{0\}} G(x)
$$

for the Polya constants. For $d \geq 3$, by Jain-Pruitt Theorem it is also possible to write $a$ in terms of the $G(x)$ function as well [32]:

$$
a=\sum_{x \in \mathbb{Z}^{d} \backslash\{0\}} \frac{\left(1-C_{d}\right)^{4} G(x)^{3}}{1+\left(1-C_{d}\right) G(x)}
$$

Then, from the convergence condition of a generic HLS urn $C_{d}=\pi\left(C_{d}\right)$ (see [7]) follows that

$$
a_{R P}=C_{d}\left(1-b_{R P}\right) .
$$



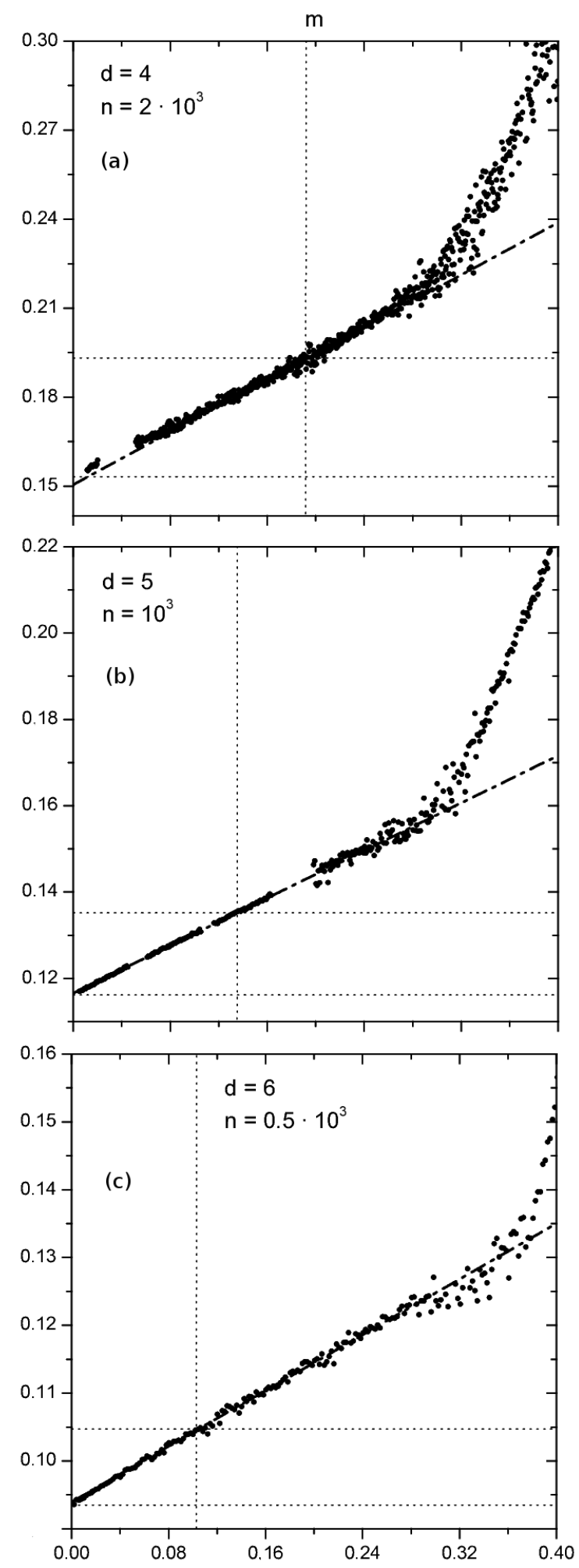

Figure 2. Numerical estimation of $\pi_{N}(\lfloor N m\rfloor)$ from Eq. [17), for $d=4,5,6$ and $0 \leq m \leq 0.4$. As for $d=3$, the lengths of the chains were $N=2000$ for $d=4, N=1000$ for $d=5$ and $N=500$ for $d=6$. For each plot, the vertical and the upper horizontal dotted lines are Polya constants $C_{d}$ [9, 16, while the lower horizontal dot lines are $\pi_{\infty}(0)=\eta_{d}$ from [17, 18]. The dash dotted lines are $\pi_{M F}(m)=C_{d}\left(1-B_{d}\right)+B_{d} m$ of Eq. 28) and 29) (not linear fits). 


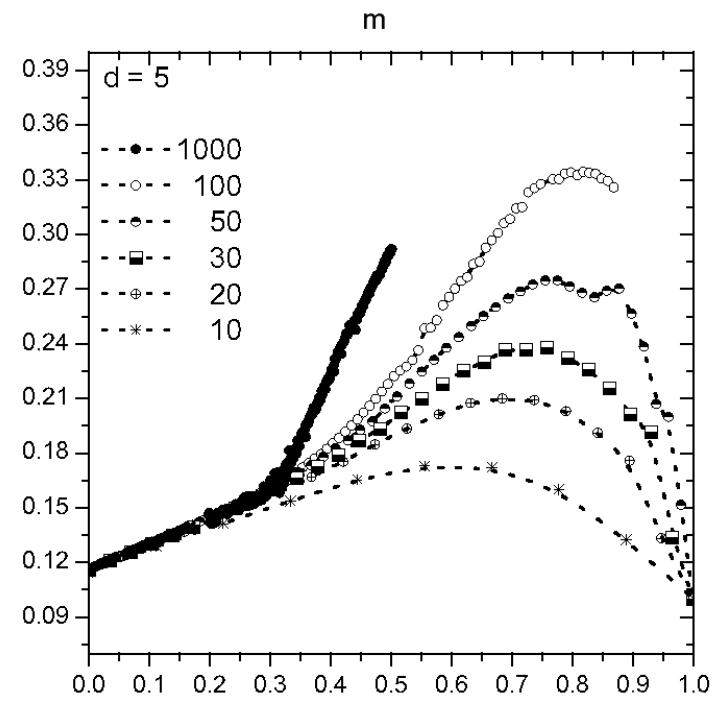

Figure 3. Numerical estimation of $\pi_{N}(\lfloor N m\rfloor)$ from Eq. 17$]$ for $d=5,0 \leq m \leq 1$ and from $N=10$ to $N=1000$. The convergence to some limit urn function $\pi(m)$ is observed in the SAW region $m<m_{c}$.

By computing the variance of the linear urn from the CGF of Eq. (11) and confronting with the expression of the $\mathrm{RP}$ variance from Jain-Pruitt Theorem above we get

$$
b_{R P}=\frac{1}{2}\left(1-\frac{C_{d}\left(1-C_{d}\right)}{\sigma_{d}^{2}}\right) .
$$

Linear urns with the above values are shown as dot lines in Figures 1 and 2. A detailed computation will be presented elsewhere.

\section{TWO COLORS MEAN-FIELD THEORY}

Besides the computational advantages in numerically studying the atmosphere instead of counting the number of walks, that has been already exploited in [13, the urn theory allows for new interesting analytic approaches. For example, here we give a simple model that match the linear urn theory suggested by our numerical simulations. In the spirit of the classic Pincus-De Gennes blob picture [14] let slice the chain $\omega_{N}$ into a number $n$ of sub-chains

$$
\omega_{N}=\left\{\omega_{T}^{0}, \omega_{T}^{1}, \ldots, \omega_{T}^{n}\right\}
$$

each of size $T=N / n$. The sub-chains are indicated with

$$
\omega_{T}^{i}=\left\{x_{0}^{i}, x_{1}^{i}, \ldots, x_{T}^{i}\right\} \subset \omega_{N}
$$

and satisfy the chain constraint

$$
x_{T}^{i}=x_{0}^{i+1} .
$$

If we neglect the mutual self-intersections between different blocks we can approximate the energy with

$$
H\left(\omega_{N}\right) \simeq \sum_{i=1}^{n} H\left(\omega_{T}^{i}\right),
$$

and the energy increment

$$
\delta H_{1}\left(\omega_{N}\right) \simeq \delta H_{1}\left(\omega_{T}^{1}\right) .
$$

The probability measure conditioned to $H\left(\omega_{N}\right)=\lfloor N m\rfloor$ is then approximated by a product measure

$$
\mu_{m}\left(\omega_{N}\right) \simeq \prod_{i=1}^{n} \mu_{m_{i}}\left(\omega_{N}^{i}\right) .
$$




\begin{tabular}{|c|c||c|c|c|}
\hline$d$ & $b_{R P}$ & $\eta_{d}^{(u)}$ & $\eta_{d}$ & $\eta_{p}^{(u)} / \eta_{d}-1$ \\
\hline \hline 3 & $1 / 2$ & $0.17026(9)$ & $0.2193(5)$ & $-22.38 \%$ \\
\hline 4 & $0.22080(9)$ & $0.15054(1)$ & $0.1532445(6)$ & $-1.76 \%$ \\
\hline 5 & $0.13767(2)$ & $0.11656(8)$ & $0.1161456(3)$ & $0.36 \%$ \\
\hline 6 & $0.10266(0)$ & $0.09396(5)$ & $0.0934921(3)$ & $0.51 \%$ \\
\hline 7 & $0.08291(2)$ & $0.078727(3)$ & $0.07837021(4)$ & $0.46 \%$ \\
\hline 8 & $0.07030(6)$ & $0.067786(5)$ & $0.0675464(2)$ & $0.36 \%$ \\
\hline
\end{tabular}

Table I. In table is shown $\eta_{d}^{(u)}=C_{d}\left(1-b_{R P}\right)$ from MF theory and numerically determined $\eta_{d}$ from literature [17, 19] 21]: while $d=3$ there is a heavy underestimation (more that 20\%), yet for $d=4$ there is error of $2 \%$, and $d \geq 5$ under the percent. An exhaustive analysis of our results about the range problem will be published in a dedicated paper.

Notice that the approximation of Eq.s (33), (34) is expected to hold at least if both $N, T \rightarrow \infty$ and $d \geq 4$ because above the critical dimension the interaction between different subwalks is negligible in the thermodynamic limit 8 [10, 17. If instead we take $T$ to be finite then the mutual intersections between the segments are no longer negligible, nonetheless, since the typical length between two self intersection is of order $O(1 / 2 d)$ we expect that the above linear approximation will be asymptotically exact also for $T<\infty$ in the limit $d \rightarrow \infty$.

Now, we approximate by assuming that the sub-chains distributions can be of two kind only, say $A$ and $B$

$$
\mu_{i}\left(\omega_{N}^{i}\right)=\varphi^{i} \mu_{A}\left(\omega_{N}^{i}\right)+\left(1-\varphi^{i}\right) \mu_{B}\left(\omega_{N}^{i}\right) .
$$

This recall again the two colors approximation, and seems a crucial technical point to obtain linear urns. We can give a simple physical understanding of this by taking $A$ to be, for example, a Self-Avoiding Walk $m_{A}=0, T$ equal to the average number of steps a SRW can do without self-intersectiong, and $B$ to contain a self-intersection such that the local range density is $1-m_{B}$ with $m_{B}=C_{d}>0$. Forcing a self-intersection in one block will certainly bring to a decrease in the total range density, on the other side this will affect the atmosphere only if the self-intersection happens near the the endpoint where we are supposed to grow the chains.

In the previous formula Eq. (36) we introduced a binary sequence

$$
\varphi=\left\{\varphi^{1}, \ldots, \varphi^{n}\right\}
$$

with $\varphi^{i} \in\{0,1\}$, that keep record of weather a subchain is either of one kind or the other, and can be interpreted as the color of the ball we add. For a walk in a given state we assume that the range density is peaked around some value

$$
H\left(\omega_{T}^{i}\right) \simeq m_{A} T \varphi^{i}+m_{B} T\left(1-\varphi^{i}\right)
$$

concerning the energy and

$$
\delta H_{1}\left(\omega_{N}\right) \simeq \pi_{B}+\left(\pi_{A}-\pi_{B}\right) \varphi^{1}
$$

for the energy increment. Given this we find

$$
H\left(\omega_{N}\right) / N \simeq m_{B}+\left(m_{A}-m_{B}\right) \frac{1}{n} \sum_{i=1}^{n} \varphi^{i},
$$

then, taking the average over $C_{N}(M)$ with

$$
\left\langle\varphi^{1}\right\rangle_{C_{N}(M)}=\frac{1}{n} \sum_{i=1}^{n}\left\langle\varphi^{i}\right\rangle_{C_{N}(M)}
$$

we arrive to a linear expression for the urn function

$$
\pi_{N}(M) \simeq a_{R P}+b_{R P} M / N,
$$

with coefficients $a_{R P}=\pi_{B}-m_{B} b_{R P}$ and

$$
b_{R P}=\frac{\pi_{A}-\pi_{B}}{m_{A}-m_{B}} .
$$

There are various ways to obtain these coefficients from Random Walks Theory. If we take $A$ to be the RW and $B$ to be the SAW, we arrive to the linear urn described before, where $a_{R P}$ equals the SAW normalized connective 
constant $\eta_{d}$ [17, 18, and $b_{R P}=1-\eta_{d} / C_{d}$. By comparing to mean-field value we obtain an expression for the rescaled connective constant of the Self-Avoiding Walk [17, 18]

$$
\eta_{d} \simeq \frac{C_{d}}{2}\left(1+\frac{C_{d}\left(1-C_{d}\right)}{\sigma_{d}^{2}}\right)=\eta_{d}^{(u)},
$$

A computation of $\eta_{d}^{(u)}$ via numerical integration (see Table I) suggests to exclude that this is the correct value for $\eta_{d}$, at least for $d \leq 8$, although our numerical analysis show narrow discrepancies as $d$ increases.

We conclude by remarking that in the above mean field theory a critical ingredient is to assume that we can obtain the urn function via interpolation between any two fixed energy states, for example sub-chains that are either selfavoiding or critically collapsed, or between self avoiding chain and random chains as well. The reason for this to hold so well in high dimensions is not clear.

The numerical estimates $\eta_{d}^{(u)}$ in Table 1, based on the linear urn analogy and the Jain-Puitt theorem, seems to indicate that Eq. (38) is slightly deviating from the accurate numerical values available in literature, at least for $d \leq 8$. Unfortunately, we expect this simple linear urn analogy to be only asymptotic for $d \rightarrow \infty$, but we also expect that more refined estimate of the urn function can be obtained by a proper accounting of mutual self intersections between the blocks. Further investigations on this aspect would be of certain interest, also, it would be interesting to understand the meaning of higher order polynomial urn functions. We expect that some light on this may be obtained at least in $d=4$ by confronting with a recently developed exact renormalization scheme based on Lace expansion [33].

\section{ACKNOWLEDGMENTS}

We would like to thank Giorgio Parisi (Sapienza Univeristà di Roma), Jack F. Douglas (NIST), Pietro Caputo (Università Roma Tre) and Valerio Paladino (Amadeus IT) for interesting discussions and suggestions. This project has received funding from the European Research Council (ERC) under the European Union's Horizon 2020 research and innovation programme (grant agreement No [694925]).

[1] L. Johnson, S. Kotz, Wiley (1977).

[2] R. Pemantle, Prob. Surv. 4 (2007), 1-79.

[3] H .M . Mahmoud, Polya Urn Models, Taylor \& Francis (2008).

[4] B. M. Hill, D. Lane, W. Sudderth, Ann. Prob. 8 (1980), 214-226.

[5] B. M. Hill, D. Lane, W. Ann. Prob. 15 (1987), 1586-1592.

[6] P. Flajolet, J. Gabarro, H. Pekari, Ann. Prob. 33 (2005), 1200-1233.

[7] S. Franchini, Stoc. Proc. Appl. 127 (2017).

[8] F. Spitzer, Principles of Random Walk (Springer, New York, 2001).

[9] B. D. Hughes, Random Walks and Random Enviroments, Vol.1 (Clarendon Press, Oxford, 1995).

[10] W. Feller, An introduction to Probability Theory and Its Applications, Vol. 1 (Wiley, New York, 1950).

[11] H. E. Stanley, K. Kang, S. Redner and R. L. Blumberg, Phys. Rev. Lett. 51, 1223 (1983); 54, 1209(E) (1985).

[12] S. Franchini, Phys. Rev. E 84, 051104 (2011).

[13] A. Rechnitzer, J. van Rensburg, J. Phys. A 84, L605 (2002).

[14] P. G. de Gennes, Scaling Concepts in Polymer Physics (Cornell University Press, 1979).

[15] P. J. Flory, Principles of Polymer Chemistry (Cornell University Press, 1971).

[16] J. F. Douglas and T. Ishinabe, Phys. Rev. E 51, 1791 (1995).

[17] N. Madras and G. Slade, The Self-Avoiding Walk (Birkhauser, Boston, 1996).

[18] It can be shown that $\pi(0)=\eta_{d}=1-\mu_{d} / 2 d$, where $\mu_{d}$ is the connective constant of the Self-Avoiding Walk [17. Also, it is possible to compute the first derivative of $\pi$ for $m=C_{d}$ exactly [28, 29]. This topic will be treated in detail elsewhere.

[19] N. Clisby, Phys. Rev. Lett. 104, 055702 (2010).

[20] N. Clisby, J. Phys. A: Math. Theor. 34, 5773 (2013).

[21] A L Owczarek and T Prellberg, J. Phys A 41, 37 (2008).

[22] M. N. Rosenbluth and A. W. Rosenbluth, J. Chem. Phys. 23, 356 (1955).

[23] The Stanley Model (SM, see [1] for the original formulation, or 9] for a review) is a model of correlated random walks which describes polymer chains with either repulsion or attractions. The attractive case is mathematically equivalent to the Rosenstock Trapping Model (random walk on lattice with randomly distributed irreversible traps: a walker encountering a trap is killed there, see [9]).

[24] Our simulations have been performed by a standard implementation of the Pruned-Enriched Rosenbluth Method (PERM), a stochastic growth algorithm that combines the Rosenbluth-Rosenbluth method with recursive enrichment. One starts by building instances according to a biased distribution, but corrects for this by cloning desired (enriching) and killing 
undesired configurations (pruning) to contain the weights fluctuations of the samples: see [25]27] for reviews and 25] for a pseudocode.

[25] P. Grassberger, Phys. Rev. E 56, 3682 (1997).

[26] T. Prellberg and J. Krawczyk, Phys. Rev. Lett. 92, 120602 (2004).

[27] H.-P. Hsu and P. Grassberger, J. Stat. Phys. 144, 597 (2011).

[28] N. C. Jain and W. E. Pruitt, J. Analyse Math. 24, 369 (1971).

[29] N. C. Jain, S. Orey, Isr. J. Math. 6, 373 (1968).

[30] A. Dvoretzky and P. Erdos, Proc. 2nd Berkley Symp. on Prob. and Stat., 353 (1951).

[31] D. C. Torny, J. Stat. Phys. 44, 49 (1986).

[32] F. Den Hollander, J. Stat. Phys. 37 (1984) 331-367.

[33] D.C. Brydges and G. Slade, J. Stat. Phys. 159 (2015) 421-667. 